\title{
L'industria dell'evidenza scientifica e la salute del metodo galileiano
}

\author{
Fulvio Floccari \\ U.O.C. Nefrologia e Dialisi, Ospedale S. Paolo, Civitavecchia (RM)
}

\begin{abstract}
The industry of scientific evidence and the health of Galilean method
Starting from the recent case of Obokata et al published on Nature, here is a raw, ruthless analysis of many possible distortions in the world of scientific publishing.

From the market of paper reprints to the lobby of editors and editorial boards, the intellectual honesty is still present in the world of literature?
\end{abstract}

Keywords: Scientific publishing, Editors, Editorial boards, Intellectual honesty

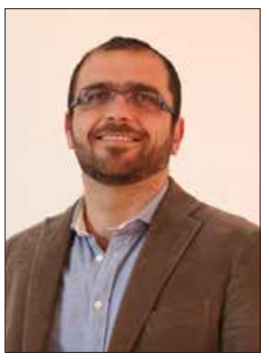

Fulvio Floccari

\section{Un gesto assurdo}

Scalare le vette della comunità scientifica anche se al prezzo del tradimento verso la propria onestà intellettuale.

Duccio Lombardi sparge sale su una ferita aperta nella storia della letteratura scientifica: gli articoli "Stimulus-triggered fate conversion of somatic cells into pluripotency" (1) e "Bidirectional developmental potential in reprogrammed cells with acquired pluripotency" (2), apparsi nel Gennaio del 2014 sulla prestigiosa rivista Nature.

Due casi eclatanti, certo, ma avvolti dal dubbio di essere solo i più recenti e clamorosi di una lunga schiera di pubblicazioni, i cui risultati sarebbero frutto di manipolazione dei dati, ad opera degli Autori.

Non riassumo qui la vicenda, che Duccio vi presenta nel suo articolo, ma mi permetto di fare alcune riflessioni, pur consapevole del rischio di risultare moralista.

Accepted: June 10, 2015

Published online: June 16, 2015

Indirizzo per la corrispondenza:

Dr. Fulvio Floccari

U.O.C. Nefrologia e Dialisi

Ospedale S. Paolo

Largo Donatori di Sangue 1

00053 Civitavecchia (RM)

fulviofloccari@gmail.com
Il primo aspetto da affrontare è quello dell'assurdità del gesto.

Una metodica di laboratorio rivoluzionaria, che certamente è destinata a finire sotto l'attenzione di tanti ricercatori nel tentativo di replicarla e farne la base per nuove pubblicazioni scientifiche, come può essere costruita senza il più elevato rigore scientifico? Quanto a lungo si spera che la menzogna possa durare? È evidente che chi si è reso protagonista di questo gesto era evidentemente accecato dal desiderio di pubblicare su una rivista tanto prestigiosa da non ponderare attentamente gli effetti del proprio gesto e la pioggia di smentite che sarebbe arrivata sul suo lavoro.

\section{Pubblicazioni "a pagamento"}

Il mondo dell'editoria scientifica ha subito negli anni un radicale cambiamento. Centinaia di riviste sono sorte in ogni campo dello scibile medico, molte delle quali trovano sostentamento non nel numero di copie vendute o nella propria capacità di attrarre investimenti pubblicitari, ma nel pagamento di un contributo proprio da parte di chi sottopone la propria opera per la pubblicazione (una cosiddetta "submission fee", che dovrebbe coprire i costi di gestione dell'opera di peer review, in realtà svolta dai referee a titolo gratuito). Un'industria scientifica di base sempre più spesso in Paesi come il Pakistan e l'India o nel continente africano e che spessissimo garantisce l'accesso gratuito e solo on line ai propri lettori.

È un paradosso per il quale non è chi cerca le informazioni a pagare il servizio, ma anche (e soprattutto) chi 
intende trasmetterle. Molte di queste riviste offrono addirittura la possibilità di scegliere una rosa di "esperti" che curino la revisione del manoscritto e lo giudichino o meno pubblicabile. II sistema della selezione peer review viene così corrotto e piegato al desiderio di "dopare" i curricula e gonfiarli con poca fatica e minor rispetto del rigore scientifico.

\section{Il fenomeno delle reprint}

Altro aspetto preoccupante è poi la dinamica commerciale delle cosiddette "reprint", ristampe in varie lingue, sponsorizzate dall'industria farmaceutica che le usa per diffondere la "medicina basata sulle evidenze" e che entrano pesantemente nel bilancio della casa editoriale, tanto da rendere legittimo il dubbio che l'Editor della rivista possa, in qualche misura, essere condizionato nella scelta se pubblicare o meno il grande trial internazionale.

\section{Editori e comitati di redazione}

Il mondo degli Editor delle riviste più prestigiose è un'oligarchia. Lo stesso grande nome della materia termina il proprio mandato presso una rivista e subito trova posto in un'altra affine, in un valzer di poltrone che raramente lascia spazio al ricambio. Lo stesso vale per gli Editorial Board delle medesime riviste, popolati da alcune decine di nomi presenti con sostanziale uniformità in tutte le testate. Non è raro che lo stesso Autore che pubblica su una rivista sia contemporaneamente presente nel Board della stessa, in un conflitto di interessi che non viene dichiarato in alcun caso.

\section{La soluzione a tutti i mali}

A seguito di questa analisi volutamente spietata dei mali del mondo dell'editoria scientifica mi si potrebbe tacciare di pessimismo cosmico, magari citando la volpe di Esopo.

Permettetemi allora di attrezzare un'opportuna linea difensiva a facili attacchi di tal genere: la soluzione a questi mali si chiama spirito critico. Sviluppare la capacità di "critical appraisal" è lo strumento di difesa democratica della cultura scientifica. Imparare a leggere i materiali e i metodi di uno studio, applicando le regole del buon senso e della cultura medica prima di quelle della metodologia statistica, ci rende liberi dai condizionamenti della letteratura scientifica di scarsa qualità. I titoli e gli abstract servano a sollevare i dubbi e non a trangugiare conclusioni precotte, che solo una lettura critica del lavoro può smascherare.

Lunga vita a chi ha testato i lavori di Obokata et al. e ha trovato il coraggio di esporsi davanti a una rivista tanto blasonata quanto Nature. È grazie a uomini di scienza di tal genere che possiamo certificare che il metodo galileiano gode ancora di ottima salute.

\section{Disclosures}

Financial support: No financial support was received for this submission.

Conflict of interest: The author has no conflict of interest.

\section{Bibliografia}

1. Obokata H, Wakayama T, Sasai $\mathrm{Y}$, et al. Stimulus-triggered fate conversion of somatic cells into pluripotency. Nature 2014;505(7485):641-7. Retracted.

2. Obokata H, Sasai $\mathrm{Y}$, Niwa H, et al. Bidirectional developmental potential in reprogrammed cells with acquired pluripotency. Nature 2014;505(7485):676-80. Retracted. 